УДК 517.532

\title{
Axisymmetric Thermocapillary Motion in a Cylinder at Small Marangoni Number
}

\author{
Evgeniy P. Magdenko* \\ Institute of Computational Modeling SB RAS \\ Akademgorodok, 50/44, Krasnoyarsk, 660036
}

Russia

Received 21.04.2015, received in revised form 05.05.2015, accepted 20.06.2015

\begin{abstract}
The solution to the linear problem of axisymmetric thermocapillary motion of two non-miscible viscous fluids in a cylindrical tube is presented. Their common interface is fixed and undeformable. This problem is an inverse problem because pressure gradients are unknown functions. The solution of the non-stationary problem is presented in the form of analytical expressions. They are obtained with the use of the method of Laplace transformation. If the wall temperature is stabilized then the general solution tends to the stationary solution as time increases. Numerical calculations confirm the theoretical results.
\end{abstract}

Keywords: thermocapillary motion, inverse problem, Laplace transform, fluid-fluid interface.

DOI: 10.17516/1997-1397-2015-8-3-303-311

\section{Problem statement}

The axisymmetric motion of viscous thermally conducting fluid in a cylindrical system of coordinates is described by the Navier-Stocks equations

$$
\begin{gathered}
u_{1 t}+u_{1} u_{1 r}+v_{1} u_{1 z}+\frac{1}{\rho} p_{r}=\nu\left(\Delta u_{1}-\frac{u_{1}}{r^{2}}\right), \\
v_{1 t}+u_{1} v_{1 r}+v_{1} v_{1 z}+\frac{1}{\rho} p_{z}=\nu \Delta v_{1}, \\
u_{1 r}+\frac{1}{r} u_{1}+v_{1 z}=0, \\
\theta_{t}+u_{1} \theta_{r}+v_{1} \theta_{z}=\chi \Delta \theta,
\end{gathered}
$$

where $u_{1}(r, z, t), v_{1}(r, z, t)$ are the projections the velocity vector on the axes $r, z ; p(r, z, t)$ is the pressure; $\theta(r, z, t)$ is the deviation of the temperature from the equilibrium value; $\Delta=\partial^{2} / \partial r^{2}+r^{-1} \partial / \partial r+\partial^{2} / \partial z^{2}$ is the Laplace operator, $\rho, \nu, \chi$ are density, kinematic viscosity and thermal diffusivity, respectively.

System of equtions (1.1)-(1.4) admits of subgroup of four-dimensional continuous transformations [1]. They are generated by the operators $\left\langle\partial_{z}, t \partial_{z}+\partial_{v_{1}}, \partial_{p}, \partial_{\theta}\right\rangle$. Their invariants are $t$, $r, u$. Therefore, partially invariant solutions of rank 2 and 3 should be sought in the form [2]

$$
u_{1}=u_{1}(r, t), \quad v_{1}=v_{1}(r, z, t), \quad p=p(r, z, t), \quad \theta=\theta(r, z, t) .
$$

*magdenko_evgeniy@icm.krasn.ru

(c) Siberian Federal University. All rights reserved 
In this case, it follows from the equation of conservation of mass (1.3) that $v_{1}$ is a linear function of $z$ :

$$
v_{1}=w(r, t) z+w_{1}(r, t)
$$

Moreover, we have

$$
r w+\left(u_{1} r\right)_{r}=0 .
$$

The momentum equations (1.1), (1.2) give us the following relations

$$
\begin{gathered}
w_{t}+u_{1} w_{r}+w^{2}=\nu\left(w_{r r}+\frac{1}{r} w_{r}\right)+h(t), \\
\frac{1}{\rho} p=l(t)-\nu w_{r}-\frac{u_{1}^{2}}{2}-\frac{\partial}{\partial t} \int u_{1}(r, t) d r-\frac{h(t)}{2} z^{2}
\end{gathered}
$$

with arbitrary functions $h(t)$ и $l(t)$.

We use solutions (1.5)-(1.9) to describe the motion in a cylindrical tube of radius $b$ with the fluid-fluid interface at $a<b$. We assume for simplicity that $w_{1}=0$ in (1.6). If to write down the problem in dimensionless form, then the nonlinear term will stand Marangoni number $\mathrm{M}=æ \theta a^{2} / \rho \nu \chi$. It is assumed that $\mathrm{M} \ll 1$, that is last performed in thin layers or a very high viscosities. As a result, we obtain the following problem

$$
\begin{gathered}
w_{1 t}=\nu_{1}\left(w_{1 r r}+\frac{1}{r} w_{1 r}\right)+h_{1}(t), \quad 0<r<a, \\
w_{2 t}=\nu_{2}\left(w_{2 r r}+\frac{1}{r} w_{2 r}\right)+h_{2}(t), \quad a<r<b, \\
w_{1}(r, 0)=w_{10}(r), \quad w_{2}(r, 0)=w_{20}(r), \\
w_{1}(a, t)=w_{2}(a, t), \quad\left(\mu_{2} w_{2 r}-\mu_{1} w_{1 r}\right) z=-\frac{\partial \sigma}{\partial z}, \\
\theta_{1}(a, z, t)=\theta_{2}(a, z, t),\left.\quad\left(k_{1} \frac{\partial \theta_{1}}{\partial r}-k_{2} \frac{\partial \theta_{2}}{\partial r}\right)\right|_{r=a}=0 .
\end{gathered}
$$

Assume that the surface tension linearly depends on temperature:

$$
\sigma=\sigma^{0}-æ\left(\theta-\theta_{0}\right), \quad æ=\text { const }>0 .
$$

It is obvious that for the temperature we have following representations

$$
\theta_{j}(r, z, t)=a_{j}(r, t) z^{2}+b_{j}(r, t), \quad j=1,2 .
$$

Taking into account (1.15), the second boundary condition (1.13) can be rewritten as

$$
\mu_{2} w_{2 r}(a, t)-\mu_{1} w_{1 r}(a, t)=2 æ a_{1}(a, t) .
$$

Functions $a_{j}(r, t), b_{j}(r, t)$ satisfy the following equations

$$
\begin{gathered}
a_{1 t}=\chi_{1}\left(a_{1 r r}+\frac{1}{r} a_{1 r}\right), \quad 0<r<a, \\
a_{2 t}=\chi_{2}\left(a_{2 r r}+\frac{1}{r} a_{2 r}\right), \quad a<r<b, \\
-304-
\end{gathered}
$$




$$
\begin{gathered}
b_{1 t}=\chi_{1}\left(b_{1 r r}+\frac{1}{r} b_{1 r}\right)+2 \chi_{1} a_{1}, \quad 0<r<a, \\
b_{2 t}=\chi_{1}\left(b_{2 r r}+\frac{1}{r} b_{2 r}\right)+2 \chi_{2} a_{2}, \quad a<r<b, \\
a_{j}(r, 0)=a_{j 0}(r), \quad b_{j}(r, 0)=b_{j 0}(r) .
\end{gathered}
$$

It is necessary to add conditions of the boundedness of $w_{1}(r, t), a_{1}(r, t), b_{1}(r, t)$ at $r=0$ and no-slip conditions at $r=b$ :

$$
w_{2}(b, t)=0, \quad \int_{0}^{a} r w_{1}(r, t) d r=0, \quad \int_{a}^{b} r w_{2}(r, t) d r=0 .
$$

The last two relations follow from equation (1.7). They allow us define the functions $h_{1}(t)$ and $h_{2}(t)$ if function $a_{1}(r, t)$ is known. So at first we find functions $a_{j}(r, t)$. Taking into account (1.16) and boundary conditions (1.14), we write

$$
a_{1}(a, t)=a_{2}(a, t), \quad k_{1} \frac{\partial a_{1}(a, t)}{\partial r}=k_{2} \frac{\partial a_{2}(a, t)}{\partial r} .
$$

Functions $b_{1}(r, t)$ and $b_{2}(r, t)$ admit similar conditions. In addition, on the solid wall $r=b$ the temperature is given: $\theta_{2}(b, z, t)=\alpha(t) z^{2}+\beta(t)$, where functions $\alpha(t)$ and $\beta(t)$ are known. This means that

$$
a_{2}(b, t)=\alpha(t), \quad b_{2}(b, t)=\beta(t) .
$$

\section{The stationary solution}

Let us assume that all functions do not depend on time. Then from (1.18)-(1.20), (1.24), (1.25) we find that $a_{1}^{s}=a_{2}^{s}=$ const $=\alpha^{s}$ and

$$
\begin{gathered}
w_{1}^{s}(r)=\frac{a æ \alpha^{s}}{\mu_{2}} f_{1}(\delta)\left(\frac{1}{2}-\frac{r^{2}}{a^{2}}\right), \quad \delta=\frac{a^{2}}{b^{2}}<1, \\
w_{2}^{s}(r)=\frac{2 a æ \alpha^{s}}{\mu_{2}} \frac{1}{f_{3}(\delta)}\left[\frac{(1-\delta)^{2}}{1-\delta+\delta \ln \delta} \ln \left(\frac{r}{b}\right)+1-\frac{r^{2}}{b^{2}}\right],
\end{gathered}
$$

where

$$
\begin{aligned}
f_{1}(\delta) & =\frac{2 \delta f_{2}(\delta)}{f_{3}(\delta)}, \quad f_{2}(\delta)=\frac{\delta-1}{\delta}\left[2+\frac{(1-\delta) \ln \delta}{1-\delta+\delta \ln \delta}\right] \\
f_{3}(\delta) & =\frac{(1-\delta)^{2}}{1-\delta+\delta \ln \delta}-2 \delta+2 \mu \delta f_{2}(\delta), \quad \mu=\frac{\mu_{1}}{\mu_{2}} \\
h_{1}^{s} & =\frac{4 æ \nu \delta f_{1}(\delta) \alpha^{s}}{a \rho_{2} f_{3}(\delta)}, \quad \nu=\frac{\nu_{1}}{\nu_{2}}, \quad h_{2}^{s}=\frac{8 æ \delta \alpha^{s}}{a \rho_{2} f_{3}(\delta)} .
\end{aligned}
$$

The dependence of velocity components on $r$ is given by formulas

$$
\begin{aligned}
& u_{1}^{s}=-\frac{a^{2} æ \alpha^{s}}{4 \mu_{2}}\left(\frac{r}{a}\right)\left(1-\frac{r^{2}}{a^{2}}\right), \\
& u_{2}^{s}=-\frac{a^{2} æ \alpha^{s}}{\mu_{2} f_{3}(\delta)}\left(\frac{a}{r}\right)\left\{\frac { ( 1 - \delta ) ^ { 2 } } { 2 ( 1 - \delta + \delta \operatorname { l n } \delta ) } \left[\frac{r^{2}}{a^{2}} \ln \left(\frac{\delta r^{2}}{a^{2}}\right)-\right.\right. \\
&\left.\left.-\ln \delta+1-\frac{r^{2}}{a^{2}}\right]+\frac{r^{2}}{a^{2}}-\frac{\delta}{2} \frac{r^{4}}{a^{4}}-1+\frac{\delta}{2}\right\} .
\end{aligned}
$$


Function $b_{j}^{s}(r)$ are

$$
\begin{aligned}
& b_{1}^{s}(r)=\frac{\alpha^{s} b^{2}}{2}\left[1+(1-k) \delta \ln \delta-\frac{\delta r^{2}}{a^{2}}\right]+\beta^{s}, \quad 0 \leqslant r \leqslant a, \\
& b_{2}^{s}(r)=\frac{\alpha^{s} b^{2}}{2}\left[1-\frac{\delta r^{2}}{a^{2}}+(1-k) \delta \ln \left(\frac{\delta r^{2}}{a^{2}}\right)\right]+\beta^{s}, \quad 0 \leqslant a \leqslant b,
\end{aligned}
$$

where $k=k_{1} / k_{2}$.

\section{Solution of the problem by the method of the Laplace transform}

To solve linear adjoint problems one can use the Laplace transform [3]. It is defined as follows

$$
\tilde{a}_{j}(r, s)=\int_{0}^{\infty} a_{j}(r, t) e^{-s t} d t, \quad j=1,2 .
$$

Then the problem is reduced to a boundary value problem for ordinary differential equation

$$
\begin{gathered}
\tilde{a}_{1 r r}+\frac{1}{r} \tilde{a}_{1 r}-\frac{s}{\chi_{1}} \tilde{a}_{1}=-a_{10}(r), \quad 0<r<a, \\
\tilde{a}_{2 r r}+\frac{1}{r} \tilde{a}_{2 r}-\frac{s}{\chi_{2}} \tilde{a}_{2}=-a_{20}(r), \quad a<r<b, \\
\tilde{a}_{1}(a, s)=\tilde{a}_{2}(a, s), \quad k_{1} \frac{\partial \tilde{a}_{1}(a, s)}{\partial r}=k_{2} \frac{\partial \tilde{a}_{2}(a, s)}{\partial r}, \\
\tilde{a}_{2}(b, s)=\tilde{\alpha}(s) .
\end{gathered}
$$

General solution of equations (3.2), (3.3) can be represented in the form (the condition of boundedness of $\tilde{a}_{1}$ at $r=0$ is taken into account)

$$
\begin{aligned}
& \tilde{a}_{1}(r, s)=C_{1} I_{0}\left(\sqrt{\frac{s}{\chi_{1}}} r\right)+\int_{0}^{r} \tau\left[I_{0}\left(\sqrt{\frac{s}{\chi_{1}}} \tau\right) K_{0}\left(\sqrt{\frac{s}{\chi_{1}}} r\right)-\right. \\
& \left.-I_{0}\left(\sqrt{\frac{s}{\chi_{1}}} r\right) K_{0}\left(\sqrt{\frac{s}{\chi_{1}}} \tau\right)\right] a_{10}(\tau) d \tau \\
& \tilde{a}_{2}(r, s)=C_{2} I_{0}\left(\sqrt{\frac{s}{\chi_{2}}} r\right)+C_{3} K_{0}\left(\sqrt{\frac{s}{\chi_{2}}} r\right)+\int_{a}^{r} \tau\left[I_{0}\left(\sqrt{\frac{s}{\chi_{2}}} \tau\right) K_{0}\left(\sqrt{\frac{s}{\chi_{2}}} r\right)-\right. \\
& \left.-I_{0}\left(\sqrt{\frac{s}{\chi_{2}}} r\right) K_{0}\left(\sqrt{\frac{s}{\chi_{2}}} \tau\right)\right] a_{20}(\tau) d \tau
\end{aligned}
$$

where $I_{0}(x), K_{0}(x)$ are modified Bessel functions of the 1st and 2nd kind. The quantities $C_{1}$, $C_{2}$ and $C_{3}$ are determined from boundary conditions (3.4)

$$
C_{1}=\frac{1}{\Delta}\left|\begin{array}{ccc}
f_{1}(s) & I_{0}(z) & K_{0}(z) \\
f_{2}(s) & -I_{0}(y) & -K_{0}(y) \\
f_{3}(s) & -\sqrt{\chi} I_{1}(y) & \sqrt{\chi} K_{1}(y)
\end{array}\right|
$$




$$
\begin{gathered}
C_{2}=\frac{1}{\Delta}\left|\begin{array}{ccc}
0 & f_{1}(s) & K_{0}(z) \\
I_{0}(x) & f_{2}(s) & -K_{0}(y) \\
k I_{1}(x) & f_{3}(s) & \sqrt{\chi} K_{1}(y)
\end{array}\right|, \\
C_{3}=\frac{1}{\Delta}\left|\begin{array}{ccc}
0 & I_{0}(z) & f_{1}(s) \\
I_{0}(x) & -I_{0}(y) & f_{2}(s) \\
k I_{1}(x) & -\sqrt{\chi} I_{1}(y) & f_{3}(s)
\end{array}\right|, \\
\Delta=\left|\begin{array}{ccc}
0 & I_{0}(z) & K_{0}(z) \\
I_{0}(x) & -I_{0}(y) & -K_{0}(y) \\
k I_{1}(x) & -\sqrt{\chi} I_{1}(y) & \sqrt{\chi} K_{1}(y)
\end{array}\right|,
\end{gathered}
$$

where $x=a \sqrt{s / \chi_{1}}, y=a \sqrt{s / \chi_{2}}, z=b \sqrt{s / \chi_{2}}, \chi=\chi_{1} / \chi_{2}, k=k_{1} / k_{2}$,

$$
\begin{gathered}
f_{1}(s)=\int_{a}^{b} \tau\left[I_{0}(z) K_{0}\left(\sqrt{\frac{s}{\chi_{2}}} \tau\right)-I_{0}\left(\sqrt{\frac{s}{\chi_{2}}} \tau\right) K_{0}(z)\right] a_{20}(\tau) d \tau+\tilde{\alpha}(s), \\
f_{2}(s)=-\int_{0}^{a} \tau\left[I_{0}\left(\sqrt{\frac{s}{\chi_{1}}} \tau\right) K_{0}(x)-I_{0}(x) K_{0}\left(\sqrt{\frac{s}{\chi_{1}}} \tau\right)\right] a_{10}(\tau) d \tau \\
f_{3}(s)=k \int_{0}^{a} \tau\left[I_{0}\left(\sqrt{\frac{s}{\chi_{1}}} \tau\right) K_{1}(x)+I_{1}(x) K_{0}\left(\sqrt{\frac{s}{\chi_{1}}} \tau\right)\right] a_{10}(\tau) d \tau .
\end{gathered}
$$

When $t \rightarrow 0$ we have $I_{0}(t) \sim 1+t^{2} / 4, K_{0}(t) \sim-\ln (t / 2), I_{1}(t) \sim t / 2+t^{3} / 16, K_{1}(t) \sim$ $1 / t+t \ln (t / 2) / 2$ and

$$
\Delta(s) \sim \frac{1}{y}\left\{\frac{k x y}{2} \ln \left(\frac{a}{b}\right)-\sqrt{\chi}\left[1+\frac{y^{2}}{2} \ln \left(\frac{a}{b}\right)+\frac{x^{2}+z^{2}}{4}\right]\right\} .
$$

Therefore from (3.6)-(3.8) we obtain

$$
\lim _{s \rightarrow 0} s \tilde{a}_{j}(s)=\lim _{s \rightarrow 0} s \tilde{\alpha}(s)=\alpha^{s}=\text { const. }
$$

This means that function $a_{j}(r, t)$ tends to constant value as time increases [3].

Let us turn to the definition of the functions $\tilde{w}_{j}(r, t)$. The motion arises only under the action of thermocapillary forces, that is, initial conditions (1.12) are zero: $w_{j 0}(r)=0, j=1,2$. Then for the image $\tilde{w}_{j}(r, s)$ we have the following boundary value problem

$$
\begin{gathered}
\tilde{w}_{1 r r}+\frac{1}{r} \tilde{w}_{1 r}-\frac{s}{\nu_{1}} \tilde{w}_{1}=-\frac{\tilde{h}_{1}(s)}{\nu_{1}}, \quad 0<r<a, \\
\tilde{w}_{2 r r}+\frac{1}{r} \tilde{w}_{2 r}-\frac{s}{\nu_{2}} \tilde{w}_{2}=-\frac{\tilde{h}_{2}(s)}{\nu_{2}}, \quad a<r<b, \\
\tilde{w}_{1}(a, s)=\tilde{w}_{2}(a, s), \\
\mu_{2} \tilde{w}_{2 r}(a, s)-\mu_{1} \tilde{w}_{1 r}(a, s)=2 æ \tilde{a}_{1}(a, s),
\end{gathered}
$$




$$
\tilde{w}_{2}(b, s)=0, \quad \int_{0}^{a} r \tilde{w}_{1}(r, s) d r=0, \quad \int_{a}^{b} r \tilde{w}_{2}(r, s) d r=0 .
$$

Here, the function $\tilde{a}_{1}(a, s)$ is already known from equation $(3.5)$ and $\left|\tilde{w}_{1}(0, s)\right|<\infty$.

The solution of equations $(3.11),(3.12)$ can be represented as

$$
\begin{gathered}
\tilde{w}_{1}=D_{1} I_{0}\left(\sqrt{\frac{s}{\nu_{1}}} r\right)+\frac{\tilde{h}_{1}(s)}{s} \\
\tilde{w}_{2}=D_{2} I_{0}\left(\sqrt{\frac{s}{\nu_{2}}} r\right)+D_{3} K_{0}\left(\sqrt{\frac{s}{\nu_{2}}} r\right)+\frac{\tilde{h}_{2}(s)}{s} .
\end{gathered}
$$

The boundary conditions (3.13), (3.14) and the first condition (3.15) allow us to find the values of $D_{1}, D_{2}$ and $D_{3}$ :

$$
\begin{aligned}
& D_{1}=\frac{1}{s \Delta_{1}}\left|\begin{array}{ccc}
-\tilde{h}_{2} & I_{0}\left(z_{1}\right) & K_{0}\left(z_{1}\right) \\
\tilde{h}_{2}-\tilde{h}_{1} & -I_{0}\left(y_{1}\right) & -K_{0}\left(y_{1}\right) \\
-\frac{2 æ \sqrt{\nu_{2} s}}{\mu_{2}} \tilde{a}_{1}(a, s) & -I_{1}\left(y_{1}\right) & K_{1}\left(y_{1}\right)
\end{array}\right|, \\
& D_{2}=\frac{1}{s \Delta_{1}}\left|\begin{array}{ccc}
0 & -\tilde{h}_{2} & K_{0}\left(z_{1}\right) \\
I_{0}\left(x_{1}\right) & \tilde{h}_{2}-\tilde{h}_{1} & -K_{0}\left(y_{1}\right) \\
\frac{\mu}{\sqrt{\nu}} I_{1}\left(x_{1}\right) & -\frac{2 æ \sqrt{\nu_{2} s}}{\mu_{2}} \tilde{a}_{1}(a, s) & K_{1}\left(y_{1}\right)
\end{array}\right|, \\
& D_{3}=\frac{1}{s \Delta_{1}}\left|\begin{array}{ccc}
0 & I_{0}\left(z_{1}\right) & -\tilde{h}_{2} \\
I_{0}\left(x_{1}\right) & -I_{0}\left(y_{1}\right) & \tilde{h}_{2}-\tilde{h}_{1} \\
\frac{\mu}{\sqrt{\nu}} I_{1}\left(x_{1}\right) & -I_{1}\left(y_{1}\right) & -\frac{2 æ \sqrt{\nu_{2} s}}{\mu_{2}} \tilde{a}_{1}(a, s)
\end{array}\right| \text {, } \\
& \Delta_{1}=\left|\begin{array}{ccc}
0 & I_{0}\left(z_{1}\right) & K_{0}\left(z_{1}\right) \\
I_{0}\left(x_{1}\right) & -I_{0}\left(y_{1}\right) & -K_{0}\left(y_{1}\right) \\
\frac{\mu}{\sqrt{\nu}} I_{1}\left(x_{1}\right) & -I_{1}\left(y_{1}\right) & K_{1}\left(y_{1}\right)
\end{array}\right|
\end{aligned}
$$

where $x_{1}=a \sqrt{s / \nu_{1}} ; y_{1}=a \sqrt{s / \nu_{2}} ; z_{1}=b \sqrt{s / \nu_{2}} ; \mu=\mu_{1} / \mu_{2} ; \nu=\nu_{1} / \nu_{2}$. Since [4]

$$
\begin{gathered}
\int_{0}^{a} r I_{0}\left(\sqrt{\frac{s}{\nu_{1}}} r\right) d r=\sqrt{\frac{\nu_{1}}{s}} a I_{1}\left(\sqrt{\frac{s}{\nu_{1}}} a\right), \\
\int_{a}^{b} r I_{0}\left(\sqrt{\frac{s}{\nu_{2}}} r\right) d r=\frac{\nu_{2}}{s}\left[z_{1} I_{1}\left(z_{1}\right)-y_{1} I_{1}\left(y_{1}\right)\right], \\
\int_{a}^{b} r K_{0}\left(\sqrt{\frac{s}{\nu_{2}}} r\right) d r=\frac{\nu_{2}}{s}\left[y_{1} K_{1}\left(y_{1}\right)-z_{1} K_{1}\left(z_{1}\right)\right] .
\end{gathered}
$$


Then taking into account (3.16), (3.18) and the second and third relations (3.15), we find

$$
\begin{gathered}
D_{1}=-\frac{a \tilde{h}_{1}(s)}{2 \sqrt{\nu_{1} s} I_{1}\left(x_{1}\right)}, \\
D_{2}\left[z_{1} I_{1}\left(z_{1}\right)-y_{1} I_{1}\left(y_{1}\right)\right]+D_{3}\left[y_{1} K_{1}\left(y_{1}\right)-z_{1} K_{1}\left(z_{1}\right)\right]=-\frac{\tilde{h}_{2}(s)\left(b^{2}-a^{2}\right)}{2 \nu_{2}} .
\end{gathered}
$$

Substitution of $D_{1}, D_{2}, D_{3}$ from (3.17) into (3.19) allows us to define $\tilde{h}_{1}(s)$ and $\tilde{h}_{2}(s)$ :

$$
\tilde{h}_{1}(s)=\frac{F_{1} B_{2}-A_{2} F_{2}}{A_{1} B_{2}-A_{2} B_{1}}, \quad \tilde{h}_{2}(s)=\frac{A_{1} F_{2}-F_{1} B_{1}}{A_{1} B_{2}-A_{2} B_{1}}
$$

where

$$
\begin{gathered}
A_{1}=\frac{x_{1} \Delta_{1}}{2 I_{1}\left(x_{1}\right)}+I_{0}\left(z_{1}\right) K_{1}\left(y_{1}\right)+K_{0}\left(z_{1}\right) I_{1}\left(y_{1}\right), \\
A_{2}=\frac{1}{y_{1}}-I_{0}\left(z_{1}\right) K_{1}\left(y_{1}\right)-I_{1}\left(y_{1}\right) K_{0}\left(z_{1}\right), \\
F_{1}=\frac{2 æ \tilde{a}_{1}(a, s) y_{1}}{a \rho_{2}}\left[I_{0}\left(y_{1}\right) K_{0}\left(z_{1}\right)-I_{0}\left(z_{1}\right) K_{0}\left(y_{1}\right)\right], \\
B_{1}=\frac{\mu}{\sqrt{\nu}} I_{1}\left(x_{1}\right)\left[1-y_{1} I_{1}\left(y_{1}\right) K_{0}\left(z_{1}\right)-y_{1} I_{0}\left(z_{1}\right) K_{1}\left(y_{1}\right)\right], \\
B_{2}=\left[z_{1} I_{1}\left(z_{1}\right)-y_{1} I_{1}\left(y_{1}\right)\right]\left[\frac{\mu}{\sqrt{\nu}} I_{1}\left(x_{1}\right) K_{0}\left(y_{1}\right)+I_{0}\left(x_{1}\right) K_{1}\left(y_{1}\right)-\right. \\
\left.-\frac{\mu}{\sqrt{\nu}} I_{1}\left(x_{1}\right) K_{0}\left(z_{1}\right)\right]+\left[y_{1} K_{1}\left(y_{1}\right)-z_{1} K_{1}\left(z_{1}\right)\right]\left[\frac{\mu}{\sqrt{\nu}} I_{1}\left(x_{1}\right) I_{0}\left(z_{1}\right)-\frac{\mu}{\sqrt{\nu}} I_{1}\left(x_{1}\right) I_{0}\left(y_{1}\right)\right]+\frac{(1-\delta)}{2 \delta} y_{1}^{2} \Delta_{1}, \\
F_{2}=\frac{2 æ \tilde{a}_{1}(a, s)}{a \rho_{2}} y_{1} I_{0}\left(x_{1}\right)\left[1-y_{1} K_{0}\left(z_{1}\right) I_{1}\left(y_{1}\right)-y_{1} K_{1}\left(y_{1}\right) I_{0}\left(z_{1}\right)\right] .
\end{gathered}
$$

After some complicated mathematical treatment the limiting equalities

$$
\lim _{s \rightarrow 0} s w_{j}(r, s)=w_{j}^{s}(r), \quad j=1,2
$$

are proved. This means that the solution tends to the stationary solution as time increases.

Figs. 1, 2 show the dimensionless function $\bar{w}_{j}=a^{2} w_{j} / \nu_{1}$ for silicon-water system at temperature of $20^{\circ} \mathrm{C}$. Fig. 1 presents the case when $\alpha(\tau)=\sin \left(10^{-2} \tau\right)$, where $\tau=a^{2} t / \nu_{1}-$ is the dimensionless time that is $\alpha(\tau)$ does not have limit by $\tau \rightarrow \infty$. Thus, the solution with time growth does not converge to stationary. Fig. 2 shows the case when $\alpha(\tau)=1+e^{-\tau} \sin (\tau)$.

Remark 1. The problem of determination of the image $\tilde{b}_{j}(r, s)$ is similar to problem (3.2)-(3.4). One need to replace $-a_{j 0}(r)$ with $-b_{j 0}(r)-2 \tilde{a}_{j}(r, s)$ and $\tilde{\alpha}(s)$ with $\tilde{\beta}(s)$. Thus, these functions can be found with the use of (3.5)-(3.8).

The author is grateful his scientific advisor, doctor in physical and mathematical sciences V.K.Andreev for formulating the problem and for valuable advice. The work was supported by the Russian Foundation for Basic Research, grant 14-01-0006\%. 


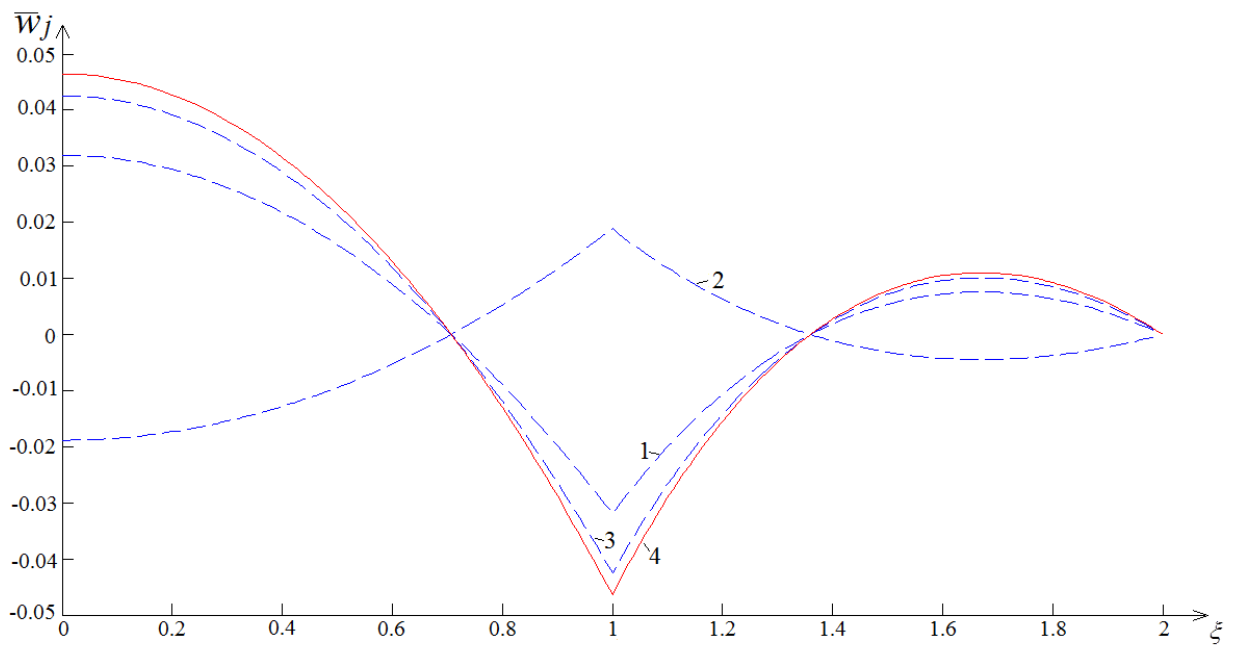

Fig. 1. Dimensionless function profiles $\bar{w}_{j}$ at $\alpha(\tau)=\sin \left(10^{-2} \tau\right)$; curve 1: $\tau=200$; curve 2: $\tau=400$; curve 3: $\tau=700$; curve 4: stationary solution. Curves $1-3$ with time growth does not converge to curve 4

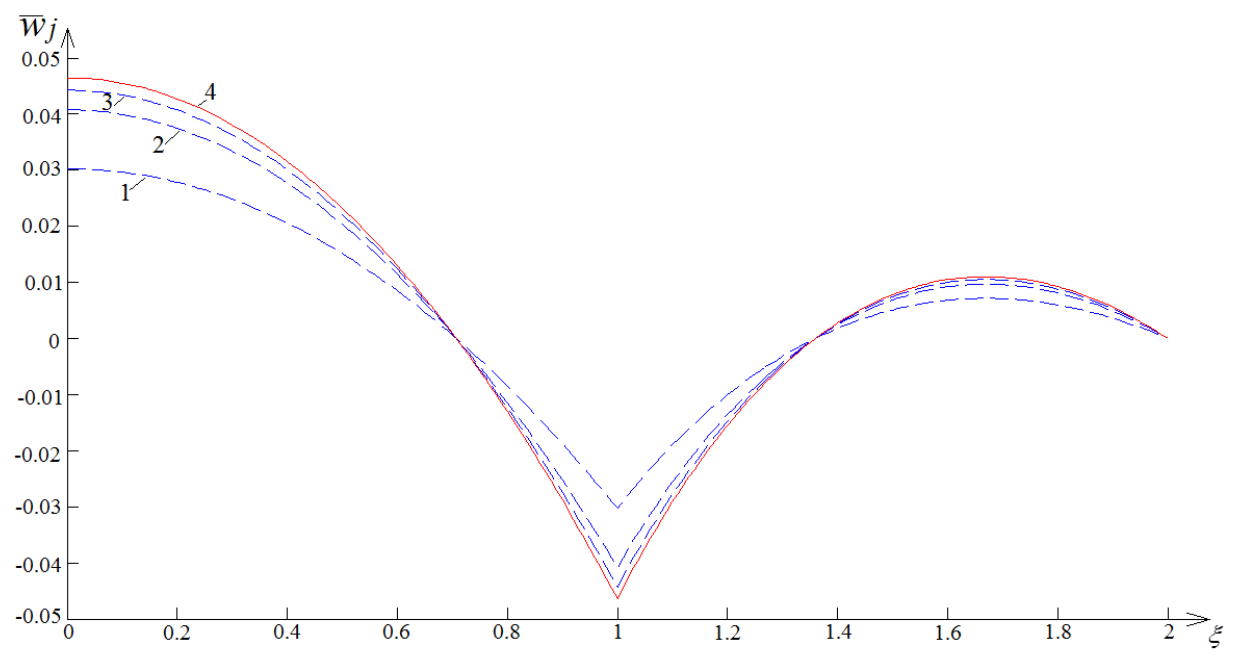

Fig. 2. Dimensionless function profiles $\bar{w}_{j}$ at $\alpha(\tau)=1+e^{-\tau} \sin (\tau)$; curve 1: $\tau=20$; curve 2: $\tau=50 ;$ curve 3: $\tau=100$; curve 4: stationary solution

\section{References}

[1] V.K.Andreev, Mathematical modeling of convective flows, study guide, Krasn. Gos. Univ., Krasnoyarsk, 2006 (in Russian).

[2] L.V.Ovsyannikov, Group analysis of differential equations, Academic Press, New York, 1982.

[3] M.A.Lavrentiev, B.V.Shabat, Methods of the theory of functions of a complex variable, 
Nauka, Moscow, 1973 (in Russian).

[4] G.Bateman, A.Erdelyi, Higher transcendental functions. 2. Bessel functions, the parabolic cylinder function, orthogonal polynomials, McGraw-Hill, 1953.

\section{Осесимметрическое термокапиллярное движение в цилиндре при малых числах Марангони}

\section{Евгений П. Магденко}

$\overline{B \text { статье решена линейная задача об осесимметрическом термокапиллярном движении двух }}$ несмешивающихся вязких теплопроводных жидкостей в иилиндрической трубе. Их общая поверхность раздела фиксируема и недеформируема. Задача является обратной, так как градиенты давлений есть искомые функиии. В изображениях по Лапласу решения находятся в виде квадратур. Доказано, что если температура на стенке трубъ стабилизируется со временем, то решение также с ростом времени стремится $к$ стационарному режиму. Проведённые численные расчёты хорошо соотносятся с теоретическими результатами.

Ключевые слова: термокапиллярность, обратная задача, преобразование Лапласа, поверхность раздела. 